\title{
Mental Health and Quality of Life for Healthcare Workers in a University Hospital Under COVID-19
}

\author{
Hye-Ji Choi ${ }^{1}$, Chan-Mo Yang ${ }^{1}$, Sang-Yeol Lee ${ }^{1}$, Hye-Jin Lee ${ }^{2}$, and Seung-Ho Jang ${ }^{1 凶}$ \\ ${ }^{1}$ Department of Psychiatry, School of Medicine, Wonkwang University, Iksan, Republic of Korea \\ ${ }^{2}$ Department of Public Health, Wonkwang University Graduate School, Iksan, Republic of Korea
}

Objective The aim of this study was to assess the psychosocial characteristics of the employees working at a university hospital and investigated the factors affecting their quality of life (QOL) under COVID-19.

Methods This study enrolled 1,191 healthcare workers from a university hospital, including doctors, nurses, administrative officer and technicians. Besides demographic information, depression, anxiety, somatization, insomnia, resilience, and QOL were assessed.

Results The nurses presented significantly higher scores for anxiety, depression and showed significantly higher insomnia scores and significantly lower resilience scores. The occupations showed significant differences in the QOL and sub-groups, including the overall quality of life and general health $(\mathrm{F}=4.774, \mathrm{p}<0.001)$, psychological domain $(\mathrm{F}=6.230, \mathrm{p}<0.001)$, and environment domain $(\mathrm{F}=5.254$, $\mathrm{p}<0.001)$. There was a positive correlation between the QOL and resilience $(\mathrm{r}=0.608, \mathrm{p}<0.01)$. However, depression $(\mathrm{r}=-0.502, \mathrm{p}<0.01)$, anxiety $(\mathrm{r}=-0.425, \mathrm{p}<0.01)$, somatization $(\mathrm{r}=-0.364, \mathrm{p}<0.01)$, and insomnia $(\mathrm{r}=-0.385, \mathrm{p}<0.01)$ showed negative correlations with the QOL. Resilience was the most important factor influencing the QOL.

Conclusion The results of this study showed that low resilience adversely affected the QOL and the mental health of the healthcare workers, which consequently had a direct effect on the quality of medical care given to patients.

Psychiatry Investig 2022;19(2):85-91

Keywords COVID-19; Mental health; Health care workers; Quality of life.

\section{INTRODUCTION}

On December 31, 2019, the Chinese health authorities reported the occurrence of pneumonia cases of an unknown cause in Wuhan city, Hubei Province, China to the World Health Organization. ${ }^{1}$ The novel coronavirus strain, with the proposed interim name "2019-nCoV" on January 7 by the WHO, was detected in the throat swab samples of the patients. ${ }^{2,3}$ This novel virus was soon renamed as severe acute respiratory syndrome coronavirus 2 (SARS-CoV-2), and the disease was officially named coronavirus disease 2019 (COVID-19) by the WHO. Furthermore, the WHO declared the SARS-CoV-2 outbreak as a public health emergency of international concern on Jan-

Received: September 16, 2021 Revised: October 1, 2021

Accepted: October 5, 2021

$\square$ Correspondence: Seung-Ho Jang, MD

Department of Psychiatry, School of Medicine, Wonkwang University, 895

Muwang-ro, Iksan 54538, Republic of Korea

Tel: +82-63-859-1044, Fax: +82-63-857-1043

E-mail: psychicjang@gmail.com

(a) This is an Open Access article distributed under the terms of the Creative Commons Attribution Non-Commercial License (https://creativecommons.org/licenses/bync/4.0) which permits unrestricted non-commercial use, distribution, and reproduction in any medium, provided the original work is properly cited. uary $30,2020{ }^{4}$

As COVID-19 continued to spread worldwide, the governments took unprecedented measures including quarantine and travel bans. China locked down the whole city, ${ }^{5}$ while Italy enforced strict measures in the entire country. ${ }^{6}$ The United States has imposed travel restrictions on non-citizens travelling from China, Iran, and most European countries. Moreover, it continues to screen travelers coming from regions severely affected by the pandemic. Despite these measures, the number of patients and mortality rate continue to rise. ${ }^{7}$

While strategies of quarantine and isolation are adopted to protect the physical health of the individual from infectious diseases, we should also consider the effect of these measures on the mental health. ${ }^{8,9}$ According to several studies, restrictive measures such as quarantine, isolation, and social distancing affect the psychological as well as emotional well-being of individuals. ${ }^{10,11}$ The psychological responses of an individual to an infectious disease include anxiety, fear, frustration, loneliness, rage, boredom, depression, stress, and maladaptive behaviors such as avoidance, emotional pain, and defensive response. ${ }^{12,13}$ Additionally, healthcare workers experience extreme stress 
due to an increasing number of confirmed and suspected cases, excessive workload, lack of personal protective equipment, exaggerated media coverage, insufficient number of supportive staff, etc. ${ }^{14}$ As reported in the studies conducted during the 2003 SARS outbreak, healthcare workers experience significant levels of stress, anxiety, and depression, as well as possible long-term psychological effects. ${ }^{15,16}$

In South Korea, the National Center for Disaster Trauma conducted a thorough mental health survey for 1,375 personnel working at public health centers at each municipality, the National Center for Mental Health, the Metropolitan Mental Health Welfare Centers, and the Basic Mental Health Promotion Centers. The research examined the presence of depression, anxiety, somatization, post-traumatic stress symptoms, suicidal risk, and resilience in the healthcare workers. According to the Report on the Mental Health Status of Disaster Psychosocial Support Personnel in 2020, among the psychological symptoms, a clinically significant level of depression was reported in $7.5 \%$ of the responders, anxiety in $4.6 \%$, somatization in $18.1 \%$, post-traumatic stress symptoms in $8.6 \%$, and suicidal risk in $0.5 \%{ }^{17}$

However, reports on abnormal mental health cases related to COVID-19 are limited, especially those on frontline healthcare workers working to overcome the disaster. Therefore, this study assessed the psychosocial characteristics of the employees working at a single university hospital and investigated the factors affecting their quality of life.

\section{METHODS}

\section{Participants}

This study enrolled 1,191 healthcare workers from a single university hospital, including doctors, nurses, administrative officer and technicians, who understood the purpose of this study and provided written informed consent. The research was conducted from November 2020 to January 2021 and was approved by the Institutional Review Board (IRB) of the Wonkwang University Hospital (IRB approval number: WKUH 2020-02-052). The protocol for the research project is conformed to the provisions of the Declaration of Helsinki.

\section{Materials and methods}

\section{Anxiety and depression}

The Hospital Anxiety and Depression Scale (HADS) developed by Zigmond and Snaith ${ }^{18}$ was used to assess the level of anxiety and depression in the participants. HADS consists of 14 items, of which the 7 odd-numbered questions represent the anxiety subscale (HAMD-A) and 7 even-numbered questions represent the depression subscale (HAMD-D). Each item is rated on a 4-point scale (range: $0-3$ ), and the optimal cutoff score is 8 for both HAMD-A and HAMD-D. In Korea, Oh et al. ${ }^{19}$ translated the scale to Korean and standardized it.

\section{Somatic symptoms}

The Patient Health Questionnaire-15 (PHQ-15), a self-administered test developed by Kroenke et al. ${ }^{20}$ to diagnose psychiatric illnesses in a primary care setting, was used to assess the somatic symptoms. The PHQ-15 comprises 15 items, and each item is rated on a 3-point scale (range: $0-2$ ). The total score reflects the severity of the somatic symptoms, where scores $\leq 5$ are defined as "low," 6-10 as "medium," and $\geq 11$ as "high." In South Korea, Han et al. ${ }^{21}$ translated it to Korean and tested its reliability and validity.

\section{Insomnia}

The Insomnia Severity Index (ISI) developed by Bastien et $\mathrm{al}^{22}$ was used to assess insomnia. It consists of total 7 items, wherein each item is rated on a scale of $0-4$, and the total score ranges between 0 and 28. The ISI assesses the insomnia severity in the past 2 weeks, degree of satisfaction with the present sleep cycle, functional impairment during the day, concerns about sleep, and diminished quality of life. In the Korean version of ISI, a total score $\geq 15$ is classified as high risk for insomnia. In South Korea, Cho et al. ${ }^{23}$ validated the Korean version of the index.

\section{Resilience}

To assess the resilience, we used the Connor-Davidson Resilience Scale (CD-RISC) developed by Conner and Davidson. ${ }^{24}$ The CD-RISC comprises 25 items, including 5 factors on hardiness, persistence, optimism, support, and spirituality in nature. Since each item is rated on a 5-point Likert scale from 0 ("not true at all") to 4 ("true nearly all of the time"), the total score ranges from 0 to 100 , with higher scores indicating greater resilience. In South Korea, Baek et al..$^{25}$ translated it to Korean and tested the validity.

\section{Quality of life}

To measure the quality of life, we used the WHO Quality of Life Instruments (WHOQOL-BREF) developed by the WHOQOL Group. ${ }^{26}$ This instrument consists of 26 items across 5 fields ( 2 questions on the Overall Quality of Life and General Health, 7 in the physical health domain, 6 in the psychological domain, 3 in the social relationships domain, and 8 in the environment domain). Each item is rated on a 5-point Likert scale from 0 ("not true at all") to 4 ("true nearly all of the time"), where a higher score reflects a better quality of life, and the optimal cut-off score is $60 .{ }^{27}$ In South Korea, Min et al. ${ }^{28}$ translated it to Korean and tested its validity and reliability. 


\section{Statistical analysis}

The demographic and psychosocial characteristics were compared among the different groups. The continuous variables are presented as means and standard deviations and categorical variables as frequencies and ratios. A one-way ANOVA was used to compare the parametric quantitative variables, followed by a Bonferroni test for post hoc analysis to further examine the differences among the groups. Pearson's correlation test was performed to assess the association between the quality of life and psychosocial factors of the participants from different occupations. To determine the factors affecting the quality of life, we performed stepwise regression analysis. All the collected data were analyzed using the Statistical Package for the Social Sciences (SPSS, version 21; IBM Corp., Armonk, NY, USA) and $p$ values $<0.05$ were considered statistically significant.

\section{RESULTS}

\section{Demographic characteristics of the study participants}

The demographic characteristics of the study participants showed that females ( $\mathrm{n}=969,81.4 \%)$, unmarried individuals $(\mathrm{n}=673,56.4 \%)$, and university graduates or above $(\mathrm{n}=761$, $63.9 \%)$ were predominant. With respect to the various occupations, the proportion of nurses $(\mathrm{n}=664,55.9 \%)$ was the largest, followed by administrative officer ( $\mathrm{n}=344,29.0 \%)$, technicians $(\mathrm{n}=129,10.9 \%)$, and doctors $(\mathrm{n}=51,4.3 \%)$ (Table 1$)$.

\section{Psychosocial characteristics according to the occupation}

The psychosocial characteristics were compared among the different occupations. The nurses presented significantly higher scores for anxiety $(6.25 \pm 3.50)$ as compared to the technicians $(4.59 \pm 3.28)$ and administrative officer $(5.03 \pm 3.27)(\mathrm{F}=$ 11.794, $\mathrm{p}<0.001)$. Similarly, the nurses showed significantly higher scores for depression $(7.97 \pm 3.69)$ as compared to the technicians $(6.50 \pm 3.87)$ and administrative officer $(6.82 \pm 3.63)$ $(\mathrm{F}=8.616, \mathrm{p}<0.001)$. The nurses $(8.47 \pm 4.70)$ and administrative officer $(6.67 \pm 4.79)$ presented higher scores for somatization than that of the doctors $(3.78 \pm 3.57)(\mathrm{F}=22.855, \mathrm{p}<0.001)$. The nurses showed significantly higher insomnia scores $(10.10 \pm 5.60)$ as compared to the doctors $(6.22 \pm 4.54)$, technicians $(7.72 \pm 5.28)$, and administrative officer $(7.55 \pm 5.27)(\mathrm{F}=18.320, \mathrm{p}<0.001)$. Furthermore, the nurses $(57.10 \pm 14.32)$ showed significantly lower resilience scores than that of the technicians $(65.82 \pm 14.09)$ and administrative officer $(60.29 \pm 14.71)(\mathrm{F}=11.254, \mathrm{p}<0.001)$ (Table 2).
Table 1. Demographic and clinical characteristics of participants

\begin{tabular}{lc}
\hline \multicolumn{1}{c}{ Variables } & Value \\
\hline Sex & $222(18.6)$ \\
Male & $969(81.4)$ \\
Female & $34.47 \pm 11.064$ \\
Age & \\
Occupation & $51(4.3)$ \\
Doctor & $664(55.9)$ \\
Nurse & $129(10.9)$ \\
Technician & $344(29.0)$ \\
Administrative officer & \\
Marital status & $673(56.4)$ \\
Single & $496(41.6)$ \\
Married & $13(1.1)$ \\
Separated & $9(0.8)$ \\
Divorced & $2(0.2)$ \\
Widowed &
\end{tabular}

\section{Characteristics of the quality of life according to the occupation}

The occupations showed significant differences in the quality of life and all its sub-groups, including the Overall Quality of Life and General Health ( $\mathrm{F}=4.774, \mathrm{p}<0.001)$, psychological domain $(\mathrm{F}=6.230, \mathrm{p}<0.001)$, and environment domain $(\mathrm{F}=$ $5.254, \mathrm{p}<0.001)$. In the post-hoc analysis of the Overall Quality of Life and General Health, the nurses $(6.17 \pm 1.35)$ showed significantly lower scores as compared to the technicians (6.67 \pm 1.46). There were significant differences in the physical health domain $(\mathrm{F}=11.931, \mathrm{p}<0.001)$ among the groups, with the nurses $(22.34 \pm 3.77)$ scoring significantly lower than the doctors (24.16 \pm 3.66$)$, technicians (24.26 \pm 4.07$)$, and administrative officer (23.42 \pm 3.70$)$. In the psychological domain, the nurses $(19.76 \pm 3.96)$ scored significantly lower than the technicians (21.26 \pm 3.52$)$. In the social relationships domain, the technicians $(10.60 \pm 3.10)$ scored significantly higher than the nurses $(9.90 \pm 1.78)$ and administrative officer $(9.86 \pm 1.76)$. In the environment domain, the nurses $(26.59 \pm 4.59)$ scored significant- 
ly lower than the technicians (28.18 \pm 4.67$)$. Moreover, the nurses $(100.73 \pm 12.49)$ scored significantly lower than the doctors $(105.69 \pm 11.28)$ and technicians $(105.88 \pm 12.21)(\mathrm{F}=6.732, \mathrm{p}<$ 0.001 ) in the total quality of life scores (Table 3 ).

\section{Correlations between the quality of life and each psychological variable}

There was a positive correlation between the quality of life and resilience $(\mathrm{r}=0.608, \mathrm{p}<0.01)$. However, depression $(\mathrm{r}=$ $-0.502, \mathrm{p}<0.01)$, anxiety $(\mathrm{r}=-0.425, \mathrm{p}<0.01)$, somatization $(\mathrm{r}=$ $-0.364, \mathrm{p}<0.01)$, and insomnia $(\mathrm{r}=-0.385, \mathrm{p}<0.01)$ showed negative correlations with the quality of life (Table 4 ).

\section{Factors affecting the quality of life}

A stepwise regression analysis was performed to determine the factors predicting the quality of life. Resilience $(\beta=0.463$, $p<0.01)$, depression $(\beta=-0.175, p<0.01)$, insomnia $(\beta=-0.141$ $\mathrm{p}<0.01)$, and anxiety $(\beta=-0.089, \mathrm{p}<0.01)$ showed statistical significance, of which resilience was the most important factor influencing the quality of life. These factors accounted for $46.0 \%$ of the explanatory variance of the total quality of life (Table 5).

\section{DISCUSSION}

As COVID-19 spread around the world, the governments implemented quarantines and travel bans on an unprecedented scale. Although the selected strategies of quarantine and isolation are adopted to protect the physical health of the individuals from infectious diseases, these measures affect their psychological as well as emotional well-being. Particularly,

Table 2. Difference of HADS, PHQ-15, ISI, and CD-RISC among the groups

\begin{tabular}{|c|c|c|c|c|c|c|}
\hline Variables & $\begin{array}{l}\text { Doctor }^{\mathrm{a}} \\
(\mathrm{N}=79)\end{array}$ & $\begin{array}{l}\text { Nurse }^{\mathrm{b}} \\
(\mathrm{N}=664)\end{array}$ & $\begin{array}{l}\text { Technician }^{\mathrm{c}} \\
(\mathrm{N}=129)\end{array}$ & $\begin{array}{l}\text { Administrative officer }^{\mathrm{d}} \\
\qquad(\mathrm{N}=344)\end{array}$ & $\mathrm{F}$ & $\begin{array}{c}\text { Post-hoc } \\
\text { Bonferroni test }\end{array}$ \\
\hline \multicolumn{7}{|l|}{ HADS } \\
\hline Anxiety & $5.25 \pm 3.46$ & $6.25 \pm 3.50$ & $4.59 \pm 3.28$ & $5.03 \pm 3.27$ & $11.794^{* * *}$ & $c, d<b$ \\
\hline Depression & $6.75 \pm 3.97$ & $7.97 \pm 3.69$ & $6.50 \pm 3.87$ & $6.82 \pm 3.63$ & $8.616^{* * *}$ & $c, d<b$ \\
\hline PHQ-15 & $3.78 \pm 3.57$ & $8.47 \pm 4.70$ & $5.70 \pm 4.50$ & $6.67 \pm 4.79$ & $22.855^{* * *}$ & $\mathrm{a}, \mathrm{c}, \mathrm{d}<\mathrm{b}, \mathrm{a}<\mathrm{d}$ \\
\hline ISI & $6.22 \pm 4.54$ & $10.10 \pm 5.60$ & $7.72 \pm 5.28$ & $7.55 \pm 5.27$ & $18.320^{* * *}$ & $a, c, d<b$ \\
\hline CD-RISC & $61.41 \pm 17.00$ & $57.10 \pm 14.32$ & $65.82 \pm 14.09$ & $60.29 \pm 14.71$ & $11.254^{* * *}$ & $c, d<b$ \\
\hline
\end{tabular}

Data are presented as mean \pm standard deviation. ${ }^{* * *} \mathrm{p}<0.001$. HADS, Hospital Anxiety Depression Scale; ISI, Insomnia severity scale; PHQ15, Patient Health Questionnaire-15; CD-RISC, Connor-Davidson Resilience Scale

Table 3. Comparison of WHOQOL-BREF among the groups

\begin{tabular}{|c|c|c|c|c|c|c|}
\hline Variables & $\begin{array}{l}\text { Doctor }^{\mathrm{a}} \\
(\mathrm{N}=79)\end{array}$ & $\begin{array}{l}\text { Nurse }^{\mathrm{b}} \\
(\mathrm{N}=664)\end{array}$ & $\begin{array}{c}\text { Technician }^{\mathrm{c}} \\
(\mathrm{N}=129)\end{array}$ & $\begin{array}{l}\text { Administrative officer }^{\mathrm{d}} \\
\qquad(\mathrm{N}=344)\end{array}$ & $\mathrm{F}$ & $\begin{array}{c}\text { Post-hoc } \\
\text { Bonferroni test }\end{array}$ \\
\hline Overall & $6.57 \pm 1.45$ & $6.17 \pm 1.35$ & $6.67 \pm 1.46$ & $6.37 \pm 1.28$ & $4.774^{* * *}$ & $\mathrm{~b}<\mathrm{c}$ \\
\hline Physical & $24.16 \pm 3.66$ & $22.34 \pm 3.77$ & $24.26 \pm 4.07$ & $23.42 \pm 3.70$ & $11.931^{* * *}$ & $\mathrm{~b}<\mathrm{a}, \mathrm{c}, \mathrm{d}$ \\
\hline Psychological & $21.24 \pm 3.42$ & $19.76 \pm 3.96$ & $21.26 \pm 3.52$ & $20.27 \pm 3.35$ & $6.230^{* * *}$ & $\mathrm{~b}<\mathrm{c}$ \\
\hline Social & $10.33 \pm 1.97$ & $9.90 \pm 1.78$ & $10.60 \pm 3.10$ & $9.86 \pm 1.76$ & $4.340^{* * *}$ & $b, d<c$ \\
\hline Environmental & $28.41 \pm .3 .71$ & $26.59 \pm 4.59$ & $28.18 \pm 4.67$ & $27.18 \pm 4.31$ & $5.254^{* * *}$ & $\mathrm{~b}<\mathrm{c}$ \\
\hline Sum of WHOQOL-BREF & $105.69 \pm 11.28$ & $100.73 \pm 12.49$ & $105.88 \pm 12.21$ & $102.58 \pm 11.35$ & $6.732^{* * *}$ & $\mathrm{~b}<\mathrm{a}, \mathrm{c}$ \\
\hline
\end{tabular}

Data are presented as mean \pm standard deviation. ${ }^{* * *} \mathrm{p}<0.001$. WHOQOL-BREF, Korean Version of World Health Organization Quality of Life Assessment Instrument Brief Form

Table 4. Bivariate associations between WHOQOL-BREF and psychological variables

\begin{tabular}{|c|c|c|c|c|c|c|}
\hline Variables & WHOQOL-BREF & HADS (anxiety) & HADS (depression) & PHQ-15 & ISI & CD-RISC \\
\hline WHOQOL-BREF & 1 & & & & & \\
\hline HADS (anxiety) & $-0.425^{*}$ & 1 & & & & \\
\hline HADS (depression) & $-0.502^{*}$ & $0.661^{*}$ & 1 & & & \\
\hline PHQ-15 & $-0.364^{*}$ & $0.586^{*}$ & $0.558^{*}$ & 1 & & \\
\hline ISI & $-0.385^{*}$ & $0.453^{*}$ & $0.451^{*}$ & $0.541^{*}$ & 1 & \\
\hline CD-RISC & $0.608^{*}$ & $-0.338^{*}$ & $-0.441^{*}$ & $-0.289^{*}$ & -0.270 & 1 \\
\hline
\end{tabular}

${ }^{*} \mathrm{p}<0.01$. WHOQOL-BREF, World health organization quality of life assessment instrument brief form; HADS, Hospital Anxiety Depression Scale; PHQ-15, Patient health questionnaire-15; ISI, Insomnia severity scale; CD-RISC, Connor-Davidson Resilience Scale 
Table 5. Stepwise regression analysis of WHOQOL-BREF among the subjects

\begin{tabular}{lcccccc}
\hline & Standardized $\beta$ & $\mathrm{t}$ & $\mathrm{p}$ & Adjusted $\mathrm{R}^{2}$ & $\mathrm{~F}$ & $\mathrm{p}$ \\
\hline CD-RISC & 0.463 & 19.425 & $<0.01$ & 0.460 & 9.255 & \\
HADS (depression) & -0.175 & -5.761 & $<0.01$ & & \\
ISI & -0.141 & -5.731 & $<0.01$ & & \\
HADS (anxiety) & -0.089 & -3.042 & $<0.01$ & & \\
\hline
\end{tabular}

WHOQOL-BREF, World health organization quality of life assessment instrument brief form; HADS, Hospital Anxiety Depression Scale; ISI, Insomnia severity scale; CD-RISC, Connor-Davidson Resilience Scale

healthcare workers suffer from extreme stress. However, only few cases of mental health problems related to COVID-19 have been reported, especially those among the frontline healthcare workers fighting against this disaster. Therefore, this study intended to assess the psychosocial characteristics of the healthcare workers at a single university hospital and investigate the factors affecting their quality of life.

As compared to the other occupation groups, the nurses demonstrated higher levels of depression, anxiety, and somatization, but lower resilience. According to previous studies, Yoon and $\mathrm{Cho}^{29}$ reported that working in shifts could disturb the circadian rhythm, sleep, and daily life. The nurses on three rotating shifts had higher stress levels than those on fixed shifts. Moreover, lack of occupational autonomy, increased workload, and inadequate recognition and compensation affected the mental health of the nurses. ${ }^{30,31}$ In the process of familiarizing themselves with new tasks, nurses may face physical and psychological stress from their interaction with the patients and their colleagues. Additionally, continuous rise in the number of patients is associated with increased job stress. ${ }^{32}$ According to a study conducted for the medical staff working in the emergency room during the SARS outbreak, ${ }^{33}$ the nurses complained of more psychological distress including depression and anxiety as compared to the doctors. Similar results were observed during the COVID-19 pandemic, which could be attributed to the longer contact time and closer proximity of the nurses with the patients than that of the doctors. ${ }^{34-36}$ Additionally, due to unfamiliar work environment, new colleagues, complex work procedures, risk of infection, and prolonged time to wear the protective equipment, the nurses may experience increased psychological burden of anxiety, depression, and fear. ${ }^{37}$

The WHO defined the quality of life as "an individual's perception of their position in life in the context of culture and value systems in which they live and in relation to their goals, expectations, standards, and concerns. ${ }^{138}$ The COVID-19 pandemic resulted in the deaths of 2 million people worldwide, and the workload of healthcare workers increased dramatically. According to previous studies, due to COVID-19, the medical staff encountered high levels of anxiety and depression, and their quality of life reduced. In this study, the quality of life showed a negative correlation with depression, anxiety, soma- tization, and sleep disturbance, but a positive correlation with resilience. ${ }^{39,40}$

Resilience, which is a part of the physical and psychological characteristics of an individual, refers to the ability to adapt to changes and cope with stressful situations. ${ }^{41}$ The results of this study showed that low resilience adversely affected the quality of life and the mental health of the healthcare workers, which consequently had a direct effect on the quality of medical care given to patients. ${ }^{42,43}$ Since resilience can be acquired and strengthened through learning, ${ }^{44}$ we believe that it is necessary to adopt educational programs and psychiatric therapy to promote the resilience of the disaster workers in South Korea. There are several ways to increase resilience, including self-management strategies such as regular exercise, ${ }^{45}$ sleep hygiene education, ${ }^{46}$ mindfulness, and meditation. ${ }^{47}$ In terms of organizational justice, reasonable distribution of work, ${ }^{45,48}$ compliance with working hours, and effective communication are recommended. ${ }^{45,49}$ By utilizing e-learning and video platforms, medical institutions can also engage in interventions to improve communication skills, case management, and troubleshooting strategies to solve any possible psychological problems that might arise when treating COVID-19 patients..$^{50}$

There are some limitations in this study. First, there is a limit to the generalization of its results since the study participants were from a single university hospital. Second, the proportion of doctors among the study participants was small. Third, as a cross-sectional study, the confounding factors including exposure level of the patients infected with COVID-19, baseline anxiety and depressive symptoms of healthcare worker, and other are not adjusted in their mental health and quality of life in COVID-19 pandemic. There are limitations in inferring the causal relationships. Nevertheless, this study is the first report on the mental health of disaster workers and the inadequacy in the current policy or system to prevent stress in disaster workers. Thus, through a mental health survey, this study sought to provide the necessary evidence for promoting the mental health of these workers, which would in turn improve the quality of medical care given to the patients.

The confounding factors including exposure level of the patients infected with COVID-19, baseline anxiety and depression symptoms of healthcare worker, and other should be 
adjusted in their mental health and quality of life in COVID-19 pandemic. If it cannot be conducted, the contents should be described in the limitation.

\section{Availability of Data and Material}

The datasets generated or analyzed during the study are available from the corresponding author on reasonable request.

\section{Conflicts of Interest}

The authors have no potential conflicts of interest to disclose.

\section{Author Contributions}

Conceptualization: Seung-Ho Jang, Hye-Ji Choi. Data curation: SeungHo Jang, Hye-Jin Lee. Formal analysis: Seung-Ho Jang, Hye-Ji Choi, ChanMo Yang. Funding acquisition: Seung-Ho Jang. Investigation: Seung-Ho Jang, Hye-Ji Choi, Sang-Yeol Lee, Hye-Jin Lee. Methodology: Seung-Ho Jang. Project administration: Seung-Ho Jang, Chan-Mo Yang. Resources: Seung-Ho Jang. Software: Seung-Ho Jang, Hye-Ji Choi, Sang-Yeol Lee, HyeJin Lee. Supervision: Seung-Ho Jang, Sang-Yeol Lee. Validation: Seung-Ho Jang. Visualization: Seung-Ho Jang, Hye-Ji Choi, Chan-Mo Yang. Wiriting-original draft: Seung-Ho Jang, Hye-Ji Choi. Writing_review \& editing: Seung-Ho Jang, Hye-Ji Choi.

\section{ORCID iDs}

Hye-Ji Choi

Chan-Mo Yang

Sang-Yeol Lee

Hye-Jin Lee

Seung-Ho Jang

https://orcid.org/0000-0001-8280-3963 https://orcid.org/0000-0002-4959-7595 https://orcid.org/0000-0003-1828-9992 https://orcid.org/0000-0002-1039-9196 https://orcid.org/0000-0002-3479-0552

\section{Funding Statement}

This study was supported by Wonkwang University in 2021.

\section{REFERENCES}

1. Esakandari H, Nabi-Afjadi M, Fakkari-Afjadi J, Farahmandian N, Miresmaeili SM, Bahreini E. A comprehensive review of COVID-19 characteristics. Biol Proced Online 2020;22:19.

2. Lu H, Stratton CW, Tang YW. Outbreak of pneumonia of unknown etiology in Wuhan, China: the mystery and the miracle. J Med Virol 2020;92:401-402.

3. Hui DS, I Azhar E, Madani TA, Ntoumi F, Kock R, Dar O, et al. The continuing 2019-nCoV epidemic threat of novel coronaviruses to global health - The latest 2019 novel coronavirus outbreak in Wuhan, China. Int J Infect Dis 2020;91:264-266.

4. Burki TK. Coronavirus in China. Lancet Respir Med 2020;8:238-238.

5. Rubin GJ, Wessely S. The psychological effects of quarantining a city. BMJ 2020;368: m313.

6. Yuan J, Li M, Lv G, Lu ZK. Monitoring transmissibility and mortality of COVID-19 in Europe. Int J Infect Dis 2020;95:311-315.

7. Parmet WE, Sinha MS. Covid-19- The law and limits of quarantine. N Engl J Med 2020;382:e28.

8. Hossain MM, Sultana A, Purohit N. Mental health outcomes of quarantine and isolation for infection prevention: a systematic umbrella review of the global evidence. Epidemiol Health 2020;42:e2020038.

9. Brooks SK, Webster RK, Smith LE, Woodland L, Wessely S, Greenberg $\mathrm{N}$, et al. The psychological impact of quarantine and how to reduce it: rapid review of the evidence. Lancet 2020;395:912-920.

10. Robertson E, Hershenfield K, Grace SL, Stewart DE. The psychosocial effects of being quarantined following exposure to SARS: a qualitative study of Toronto health care workers. Can J Psychiatry 2004;49:403407.

11. Barbisch D, Koenig KL, Shih FY. Is There a case for quarantine? Per- spectives from SARS to ebola. Disaster Med Public Health Prep 2015; 9:547-553.

12. Park JS, Lee EH, Park NR, Choi YH. Mental health of nurses working at a government-designated hospital during a MERS-CoV outbreak: a cross-sectional study. Arch Psychiatr Nurs 2018;32:2-6.

13. Jeong H, Yim HW, Song YJ, Ki M, Min JA, Cho J, et al. Mental health status of people isolated due to Middle East Respiratory Syndrome. Epidemiol Health 2016;38:e2016048.

14. Lai J, Ma S, Wang Y, Cai Z, Hu J, Wei N, et al. Factors associated with mental health outcomes among health care workers exposed to coronavirus disease 2019. JAMA Network Open 2020;3:e203976.

15. Lee AM, Wong JG, McAlonan GM, Cheung V, Cheung C, Sham PC, et al. Stress and psychological distress among SARS survivors 1 year after the outbreak. Can J Psychiatry 2007;52:233-240.

16. Lin CY, Peng YC, Wu YH, Chang J, Chan CH, Yang DY. The psychological effect of severe acute respiratory syndrome on emergency department staff. Emerg Med J 2007;24:12-17.

17. 2020 Survey on Mental Health of Disaster Psychological Support Workers. Available at: www.nct.go.kr. Accessed May 5, 2021.

18. Zigmond AS, Snaith RP. The hospital anxiety and depression scale. Acta Psychiatr Scand 1983;67:361-370.

19. Oh SM, Min KJ, Park DB. A study on the standardization of the hospital anxiety and depression scale for Koreans: a comparison of normal, depressed and anxious groups. J Korean Neuropsychiatr Assoc 1999; 38:289-296.

20. Kroenke K, Spitzer RL, Williams JB. The PHQ-15: validity of a new measure for evaluating the severity of somatic symptoms. Psychosom Med 2002;64:258-266.

21. Han C, Pae CU, Patkar AA, Masand PS, Kim KW, Joe SH, et al. Psychometric properties of the Patient Health Questionnaire-15 (PHQ$15)$ for measuring the somatic symptoms of psychiatric outpatients. Psychosomatics 2009;50:580-585.

22. Bastien $\mathrm{CH}$, Vallières A, Morin CM. Validation of the Insomnia Severity Index as an outcome measure for insomnia research. Sleep Med 2001;2:297-307.

23. Cho YW, Song ML, Morin CM. Validation of a Korean version of the insomnia severity index. J Clin Neurol 2014;10:210-215.

24. Connor KM, Davidson JR. Development of a new resilience scale: the Connor-Davidson Resilience Scale (CD-RISC). Depress Anxiety 2003; 18:76-82.

25. Baek HS, Lee KU, Joo EJ, Lee MY, Choi KS. Reliability and validity of the Korean version of the Connor-Davidson Resilience Scale (K-CDRISC). Psychiatry Investig 2010;7:109-115.

26. Development of the World Health Organization WHOQOL-BREF quality of life assessment. The WHOQOL Group. Psychol Med 1998; 28:551-558.

27. Silva PAB, Soares SM, Santos JFG, Silva LB. Cut-off point for WHOQOL-bref as a measure of quality of life of older adults. Rev Saude Publica 2014;48:390-397.

28. Min SK, Kim KI, Lee CI, Jung YC, Suh SY, Kim DK. Development of the Korean versions of WHO Quality of Life scale and WHOQOLBREF. Qual Life Res 2002;11:593-600.

29. Yoon HS, Cho YC. Relationship between job stress contents, psychosocial factors and mental health status among university hospital nurses in Korea. J Prev Med Public Health 2007;40:351-362.

30. Lee HS. Effect of the occupational stress and self esteem on mental health among nurses. J Kor Cont Assoc 2013;13:251-259.

31. Phoenix BJ. The current psychiatric mental health registered nurse workforce. J Am Psychiatr Nurses Assoc 2019;25:38-48.

32. Potter C. To what extent do nurses and physicians working within the emergency department experience burnout: a review of the literature. Aust Emerg Nurs J 2006;9:57-64.

33. Wong TW, Yau JK, Chan CL, Kwong RS, Ho SM, Lau CC, et al. The psychological impact of severe acute respiratory syndrome outbreak on healthcare workers in emergency departments and how they cope. 
Eur J Emerg Med 2005;12:13-18.

34. Lai J, Ma S, Wang Y, Cai Z, Hu J, Wei N, et al. Factors associated with mental health outcomes among health care workers exposed to coronavirus disease 2019. JAMA Netw Open 2020;3:e203976.

35. Kisely S, Warren N, McMahon L, Dalais C, Henry I, Siskind D. Occurrence, prevention, and management of the psychological effects of emerging virus outbreaks on healthcare workers: rapid review and meta-analysis. BMJ 2020;369:m1642.

36. Awano N, Oyama N, Akiyama K, Inomata M, Kuse N, Tone M, et al. Anxiety, depression, and resilience of healthcare workers in Japan during the coronavirus disease 2019 outbreak. Intern Med 2020;59:26932699.

37. Zhang Y, Wei L, Li H, Pan Y, Wang J, Li Q, et al. The psychological change process of frontline nurses caring for patients with COVID-19 during its outbreak. Issues Ment Health Nurs 2020;41:525-530.

38. Hunt SM. The problem of quality of life. Qual Life Res 1997;6:205-212.

39. Çelmeçe N, Menekay M. The effect of stress, anxiety and burnout levels of healthcare professionals caring for COVID-19 patients on their quality of life. Front Psychol 2020;11:597624.

40. Buselli R, Corsi M, Baldanzi S, Chiumiento M, Del Lupo E, Dell'Oste $\mathrm{V}$, et al. Professional quality of life and mental health outcomes among health care workers exposed to Sars-Cov-2 (Covid-19). Int J Environ Res Public Health 2020;17:6180.

41. Chan CL, Chan TH, Ng SM. The Strength-Focused and Meaning-Oriented Approach to Resilience and Transformation (SMART): a bodymind-spirit approach to trauma management. Soc Work Health Care
2006;43:9-36.

42. Robertson HD, Elliott AM, Burton C, Iversen L, Murchie P, Porteous T, et al. Resilience of primary healthcare professionals: a systematic review. Br J Gen Pract 2016;66:e423-e433.

43. Howe A, Smajdor A, Stöckl A. Towards an understanding of resilience and its relevance to medical training. Med Educ 2012;46:349-356.

44. Jackson D, Firtko A, Edenborough M. Personal resilience as a strategy for surviving and thriving in the face of workplace adversity: a literature review. J Adv Nurs 2007;60:1-9.

45. Callahan K, Christman G, Maltby L. Battling burnout: strategies for promoting physician wellness. Adv Pediatr 2018;65:1-17.

46. Lapa TA, Madeira FM, Viana JS, Pinto-Gouveia J. Burnout syndrome and wellbeing in anesthesiologists: the importance of emotion regulation strategies. Minerva Anestesiol 2017;83:191-199.

47. Mahmoud NN, Rothenberger D. From burnout to well-being: a focus on resilience. Clin Colon Rectal Surg 2019;32:415-423.

48. Kumar S. Burnout and doctors: prevalence, prevention and intervention. Healthcare (Basel) 2016;4:37.

49. Weight CJ, Sellon JL, Lessard-Anderson CR, Shanafelt TD, Olsen KD, Laskowski ER. Physical activity, quality of life, and burnout among physician trainees: the effect of a team-based, incentivized exercise program. Mayo Clin Proc 2013;88:1435-1442.

50. Chidiebere Okechukwu E, Tibaldi L, La Torre G. The impact of COVID-19 pandemic on mental health of Nurses. Clin Ter 2020;171:e399e400. 